\section{Implementation of the SELFY-MPI in five European countries: a multicenter international feasibility study}

\section{Sabrina Zora,}

Katerin Leslie Quispe Guerrero, ${ }^{1}$ Nicola Veronese, ${ }^{1}$ Alberto Ferri, ${ }^{1}$

An L.D. Boone, ${ }^{2}$ Marta Pisano Gonzalez, ${ }^{3}$ Yves-Marie Pers, ${ }^{4}$ Hein Raat, ${ }^{5}$ Graham Baker, ${ }^{6}$ Alberto Cella, ${ }^{1}$ Alberto Pilotto ${ }^{1,7}$ on behalf of the EFFICHRONIC Consortium

${ }^{1}$ Geriatrics Unit, Department of Geriatric Care, Orthogeriatrics and Rehabilitation, EO Galliera Hospital, Genova, Italy; ${ }^{2}$ FICYT Foundation for Applied Scientific Research and Technology in Asturias, Oviedo, Spain; ${ }^{3}$ SESPA, Health Service of the Principality of Asturias, Oviedo, Spain; ${ }^{4}$ Clinical Immunology and Osteoarticular Diseases Therapeutic Unit, Rheumatology Department, Lapeyronie University Hospital, Montpellier, France; ${ }^{5}$ Erasmus University Medical Center, Rotterdam, The Netherlands; ' ${ }^{6}$ ismet, Portsmouth, United Kingdom; ${ }^{7}$ Department of Interdisciplinary Medicine, University of Bari, Italy

\section{Abstract}

It is essential for welfare systems to predict the health and care needs of people with chronic diseases. The Multidimensional Prognostic Index (MPI) proved excellent accuracy in predicting negative health outcomes. Recently, a selfadministered version of MPI (SELFY-MPI) was developed and validated in community-dwelling subjects showing an excellent agreement between the two instruments regardless of age. This is a feasibility study concerns the implementation of SELFYMPI in five European countries. The SELFY-MPI includes the self-administration of Barthel Index, Instrumental Activities of daily Living (IADL), Test Your Memory (TYM) Test, Mini Nutritional Assessment-Short Form (MNA-SF), comorbidity, number of medications, and the Gijon's Socio-Familial Evaluation Scale (SFES). A descriptive analysis was performed on the data collected. 300 subjects (mean age 62 years, range 19-88 years; male/female ratio 0.81) completed the SELFY-MPI. The mean value of the SELFY-MPI was 0.131 (range: 0.0 0.563 ) showing a significant correlation with age (Pearson coefficient $=0.373$, $\mathrm{P}<0.001)$. The mean value of the SELFYMPI filling time was 15 minutes (range: 545 minutes) showing a significant correlation between age and filling time (Pearson coefficient $=0.547, \mathrm{P}<0.001)$. The SELFYMPI is an excellent self-administered tool for comprehensive self-assessment screening of community-dwelling people at risk of physical and cognitive frailty and/or socioeconomic vulnerability.

\section{Introduction}

An accurate assessment able to predict negative health outcomes in subjects with chronic diseases is a core point for social and health care national systems. Indeed, especially among older people the prevalence of multimorbidity is very high, ${ }^{1,2}$ and a comprehensive geriatric assessment (CGA) has proven to be able to explore physical, cognitive, biological and social factors in order to determine the prognosis of frail older subjects. ${ }^{3}$

Previous studies showed that the Multidimensional Prognostic Index (MPI), a prognostic tool based on a standard CGA, had excellent accuracy in predicting negative health outcomes in different settings and clinical conditions. ${ }^{4,5}$ Several studies proved that the MPI can predict short- and long-term mortality and other negative health outcomes, ${ }^{6}$ such as hospitalization, institutionalization, admission to home care services and re-hospitalization in subjects with different chronic conditions including hearth failure, ${ }^{7}$ chronic kidney disease ${ }^{8}$ cancer, ${ }^{9}$ dementia ${ }^{10}$ and other neuropsychological disorders ${ }^{11}$ and diabetes mellitus. ${ }^{12}$

Very recently, a self-administered version of the MPI (SELFY-MPI) has been developed and validated in communitydwelling subjects in the frame of the European Union co-funded project EFFICHRONIC. ${ }^{13}$ The development of this self-assessment version was in accordance with the current trend of health status selfperception widely used in epidemiological research ${ }^{14}$ and in the patient-empowerment approach. ${ }^{15}$

Generally, frailty has been investigated only among older persons; however new information into the occurrence of frailty among younger people is crucial in order to explore latent aspects of frailty. The present study describes the implementation of the SELFY-MPI in five European countries to assess its feasibility in different cultures and heterogeneous populations.
Correspondence: Sabrina Zora, Geriatrics

Unit, Department of Geriatric Care, Orthogeriatrics and Rehabilitation, EO

Galliera Hospital, Genova, Italy.

E-mail: sabrina.zora@galliera.it

Key words: SELFY-MPI, EFFICHRONIC, comprehensive geriatric assessment; multidimensional prognostic index.

Acknowledgments:The members of the EFFICHRONIC Consortium are: Marta M Pisano Gonzalez, Raquel Vazquez Alvarez, Delia Peñacoba Maestre (Health Service of the Principality of Asturias - SESPA, Spain); An LD Boone, José Ramón Hevia Fernandez Sergio Valles Garcia (Regional Ministry of Health - Government of the Principality of Asturias - CSPA, Spain); Inés Rey and Raquel Ochoa Gonzalez (the Foundation for the Promotion in Asturias of applied Scientific Research and Technology FICYT, Spain); Yves-Marie Pers, Christian Jorgensen, Verushka Valsecchi, Rosanna Ferreira Lopez, Adrien Durand, Cristina Balaguer Fernandez, Dallal Fracso (The University Hospital Center of Montpellier CHUM, France); Graham Baker, Danni Brown and Suzanne Lucas (Qismet, Portsmouth, UK); Siok-Swan Tan, Irene Fierloos, Xuxi Zhang, Petra de Vries, Hein Raat (Erasmus MC University Medical Center, Rotterdam, The Netherlands); Alberto Pilotto, Sabrina Zora, Alberto Ferri, Alberto Cella and Alessandra Argusti (EO Galliera Hospital, Genoa, Italy); Ascensión Doñate Martínez, Laura Llop Medina and Jorge Garcés (University of Valencia, Polibienestar Research Institute - UVEG, Valencia, Spain)

Funding: funding for this study was provided by the EFFICHRONIC 738127 Project which has been cofounded from the European Union's Health Programme (2014_2010). The contents of this article are the sole responsibility of the abovementioned authors and can under no circumstances be regarded as reflecting the position of the European Union.

The funding agencies had no role in design or conduct of the study; collection, management, analysis, and interpretation of the data; and preparation, review, or approval of the article

Conflict of interest: the authors declare no conflict of interest.

Received for publication: 19 August 2019 Revision received: 21 October 2019. Accepted for publication: 7 November 2019.

This work is licensed under a Creative Commons Attribution-NonCommercial 4.0 International License (CC BY-NC 4.0).

${ }^{\circ}$ Copyright: the Author(s), 2019

Licensee PAGEPress, Italy

Geriatric Care 2019; 5:8502

doi:10.4081/gc.2019.8502 


\section{Materials and Methods}

\section{Study design and population}

This is an observational study conducted in accordance with the World Medical Association's 2008 Declaration of Helsinki, the guidelines for Good Clinical Practice, and the Strengthening the Reporting of Observational Studies in Epidemiology (STROBE) guidelines. ${ }^{16}$

This feasibility study has been performed in the context of the ongoing project/joint action 738127/EFFICHRONIC which is part of the Third EU Health Programme concerning the chronic disease challenge. The objective of the EFFICHRONIC project is to reduce the burden of chronic diseases in five European counties (France, Italy, Spain, The Netherlands and United Kingdom) providing evidence on the sustainability of health systems by implementing the Chronic Disease Self-Management Programme $(\mathrm{CDSMP})^{17}$ in these study sites with a specific focus on the health, medical, cultural and socio-economic determinants more linked with chronic diseases in Europe (http://effichronic.eu).

Multiple recruiting actions have been adopted by researchers to identify community-dwelling subjects to be enrolled in the study such as: i) outpatients admitted to clinical centers; ii) volunteers from diseasespecific patients' associations; iii) specific meetings to share information about the CDSMP to general population.

Inclusion criteria were: i) subjects living in community; ii) without acute clinical conditions; iii) who are able to understand the information provided in the local language and in the informed consent; iv) who are willing to participate in the study.

All subject records and personal information were anonymized before the statistical analysis.

This study, as part of the EFFICHRONIC project, was approved by the local Ethics Committee and it has a trial registration No. 70517103 ISRCTN registry (date of registration $26^{\text {th }}$ June 2018).

\section{The Self-Administered Multidimensional Prognostic Index (SELFY-MPI)}

The SELFY-MPI considers the following eight domains assessed through selfadministered scales.

The first two dimensions measure the functional (such as feeding, bathing, personal hygiene, dressing, fecal and urinary continence and toilet use) and mobility status (getting in and out of bed/chair, walking and going up and down the stairs) assessed through the Barthel ADL and Barthel
Mobility scale respectively. ${ }^{18}$ Both scales can be self-administered. ${ }^{19}$

The third dimension concerns the independence of instrumental activities in daily living (telephone use, grocery shopping, preparing meals, housekeeping, laundry, travel, medication, handling finances) assessed through the Lawton's Instrumental Activities of Daily Living (IADL) scale. ${ }^{20} \mathrm{~A}$ self-administered version is available also for this scale.

Cognitive status is the fourth domain investigated with the self-administered cognitive screening test: Test Your Memory (TYM). ${ }^{21}$ It is a validated test composed of 10 tasks exploring several domains such as orientation, ability to copy a sentence, semantic knowledge, calculation, verbal fluency, similarities, naming, visuospatial abilities and recall of a previous copied sentence.

The fifth dimension is nutritional status measured by the Mini Nutritional Assessment Short Form (MNA-SF) ${ }^{22}$ through its validated version. The number of medications taken regularly is the sixth domain. The seventh dimension measures the comorbidity using the Cumulative Illness Rating Scale (CIRS) ${ }^{23}$ that explores the number of severe or chronic health diseases requiring drug therapies in 13 aspects of health. Self-assessment of this scale is also possible since it is asked to report health diseases for which it is taken the pharmacological treatment.

Socioeconomic domain is the last dimension assessed through the adapted version of the self-administered Gijon's social-familiar evaluation scale (SFES). ${ }^{24}$ This scale assesses the subject's household composition, net monthly household income, living situation, social relationships and social support received. Also, the SFES is a self-administered scale.

For each domain, equally to the MPI, a tripartite hierarchy was used in order to assign 0 as no problems, 0.5 as minor problems and 1 as major problem. This scoring is based on conventional literature for each scale. The sum of these eight domains has to be divided by 8 to obtain a final SELFY-MPI risk score, its range is from 0 (=no risk of mortality) to 1 (=high risk of mortality).

\section{Statistical analysis}

Range and mean values are reported in the descriptive analysis of the examined parameters. For continuous variables, the unpaired t-test was used for the comparison of mean values while the Pearson's coefficient was evaluated as a measure of the linear correlation between couples of variables.

\section{Results}

A total of 300 subjects (mean age 62 years, range 19-88 years; male/female ratio 0.81) completed the SELFY-MPI questionnaire. As reported in Table 1, the mean value of the SELFY-MPI was 0.131 (range: 00.563 ). In detail, the 8 constituent domains of SELFY-MPI showed the following mean values: Barthel-ADL 0.98 (range: 0-38); Barthel-MOB 0.54 (range: 0-15); IADL 7,67 (range: 1-8); TYM test 45.31 (range: 12-50); MNA-SF 12.09 (range: 5 - 14); CIRS 1.31 (range: 0-6); number of medications 2.36 (range: 0-15); SFES 6.8 (range: 5-21).

We observed a statistically significant correlation between the SELFY-MPI values and age (Pearson coefficient $=0.373$, $\mathrm{P}<0.001$ ), that can explain the statistically significant difference in SELFY-MPI values between the subjects under versus over 60 years of age $(0.08 \pm 0.08$ versus $0.17 \pm 0.13$, respectively). The box plot in Figure 1 shows the statistical characteristics of SELFY-MPI values in the two age-groups.

The completion of the questionnaire required a mean time of 15 minutes, with a significant correlation between age and filling time (Pearson coefficient $=0.547$, $\mathrm{P}<0.001)$ and a statistically significant difference between the two age-groups under and over-60 years $(13.5 \pm 4.7$ versus $21.7 \pm 9.2$ minutes, respectively, $\mathrm{P}<0.001)$ (Figure 2).

\section{Discussion and Conclusions}

The aim of this study is the evaluation of the SELFY-MPI feasibility in different cul-

Table 1. Single domains explored by the SELFY-MPI Questionnaire: mean values and range.

\begin{tabular}{lcc}
\hline Item & Mean value & Range \\
Barthel - ADL & 0.98 & $0-38$ \\
Barthel - MOB & 0.54 & $0-15$ \\
\hline IADL & 7.67 & $1-8$ \\
TYM & 45.31 & $12-50$ \\
\hline MNA-SF & 12.09 & $5-14$ \\
CIRS & 1.31 & $0-6$ \\
\hline Number of medications & 2.36 & $0-15$ \\
SFES & 6.8 & $5-21$ \\
\hline SELFY-MPI score & 0.131 & $0-0.563$ \\
\hline
\end{tabular}

ADL, activities of daily living; MOB, mobility; IADL, instrumental activities of daily living; TYM, test your memory; MNA-SF, mini nutritional assessment short form; CIRS, cumulative illness rating scale; SFES, Gijon's social familial evaluation scale. 
tures and heterogeneous populations. The SELFY-MPI showed a very good feasibility in five European countries regardless of the age. Results showed its easily use replicating the appropriate filling time (mean time 15 minutes, range 5-45 minutes) reported in the previous validation study $^{13}$ (mean time 16 minutes, range: 9-36 minutes). Data demonstrated that lower and higher filling time values were related to age: younger subjects have lower filling time values compared to the older people (Figure 2).

Similarly, to the MPI, the SELFY-MPI stems to the need of developing prognostic indices that can be necessary tools to the clinician in decision-making process for the best diagnostic and therapeutic interventions..$^{25}$ This need is particularly for subjects with multiple chronic diseases and frail elderly.

Currently a self-administered tool capable of measuring the frailty is a target point for the Welfare and the Health National Systems since the self-perception of frailty provides a wider screening and an opportunity for an early diagnosis of the frailty itself. ${ }^{26}$

The strength of the SELFY-MPI relies on the consistent research developed on the MPI. Indeed, the MPI has been validated in a very large sample confirming its predictive value in subjects affected by many different chronic diseases, i.e. hearth failure, ${ }^{7}$ chronic kidney disease, ${ }^{8}$ cancer, ${ }^{9}$ dementia, ${ }^{10}$ neuropsychological disorders ${ }^{11}$ and

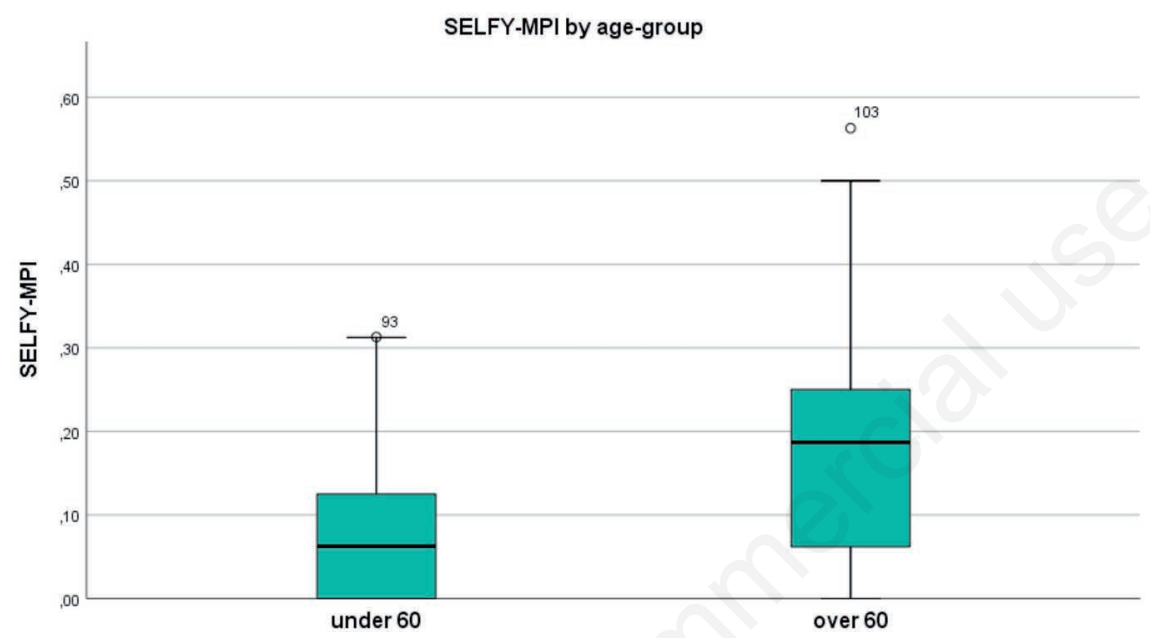

Figure 1. Mean values of the SELFY-MPI in the two age-groups: under and over 60 years $(\mathbf{P}<0.001)$.

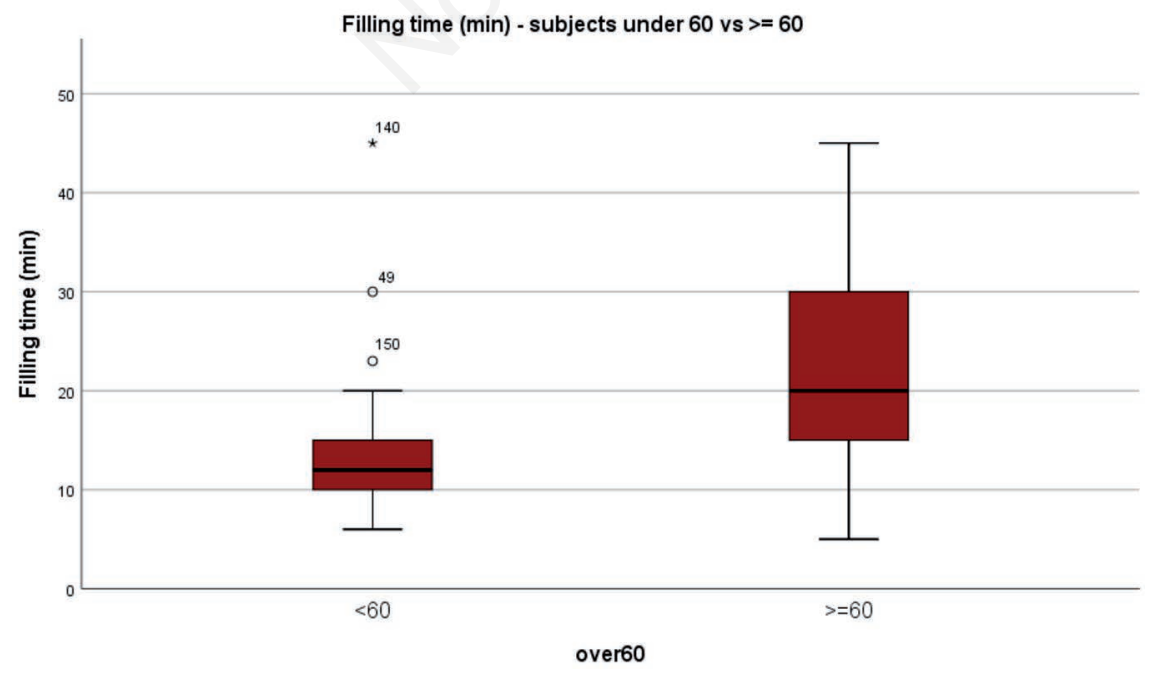

Figure 2. Mean filling times of the SELFY-MPI Questionnaire in subjects divided according to age under and over 60 years. diabetes mellitus, ${ }^{12}$ and in several settings such as in hospitalized patients, ${ }^{4,27}$ in the community-dwelling subjects who underwent a CGA to be admitted to nursing homes or homecare services, ${ }^{5}$ as well as at population level ${ }^{28}$ and at the ambulatory of the General Practitioner. ${ }^{29}$

A previous study has already showed the strong association and validity between the SELFY-MPI and the MPI. ${ }^{13}$ This study explores the feasibility of the SELFY-MPI in people of different ages and from different countries. To the best of our knowledge, the SELFY-MPI is the only self-administered tool for assessing frailty validated in 5 different languages (Dutch, English, French, Italian and Spanish) revealing its usefulness both as a screening and an outcome measure tool.

Our results showed that the study population looks quite healthy (the SELFY-MPI mean value is 0.131 ; range: $0-0.563$ ). Moreover, our sample shows low impairment in all eight constituent domains of SELFY-MPI and these data are consistent among a relatively healthy and young population. Nevertheless, these results could change if a more sick and disabled population would be enrolled. ${ }^{30}$ Therefore a selfadministered tool able to easily assess frailty like the SELFY-MPI could be a useful screening tool for both older and younger people to measure latent aspects of frailty.

It is important to take account of the limitations of this study. First, the nature of this study is cross-sectional: further longitudinal studies are required in order to verify if the SELFY-MPI can predict the outcome (mortality or negative outcomes) with the same accuracy as the MPI. Secondly, the sample enrolled is relatively small since it includes 300 subjects.

Nevertheless, there would be the opportunity in the context of the ongoing EFFICHRONIC project to assess the SELFY-MPI not only as a stratification tool but also at the 6-months follow up after the CDSMP intervention.

In conclusion, the SELFY-MPI is an excellent self-administered tool for comprehensive screening assessment of community-dwelling people at risk of physical and cognitive frailty and/or socioeconomic vulnerability. These data suggest that the SELFY-MPI could have broad applicability in subjects of different ages and from different countries.

\section{References}

1. Violan C, Foguet-Boreu Q, FloresMateo G, et al. Prevalence, determi- 
nants and patterns of multimorbidity in primary care: a systematic review of observational studies. PLoS One 2014;9:e102149.

2. Fortin M, Stewart M, Poitras M-E, et al. A systematic review of prevalence studies on multimorbidity: toward a more uniform methodology. Ann Fam Med 2012;10:142-51.

3. Pilotto A, Cella A, Pilotto A, et al. Three decades of comprehensive geriatric assessment: Evidence coming from different healthcare settings and specific clinical conditions. J Am Med Dir Assoc 2017;18:192.e1-e11.

4. Pilotto A, Ferrucci L, Franceschi M, et al. Development and validation of a multidimensional prognostic index for one-year mortality from comprehensive geriatric assessment in hospitalized older patients. Rejuvenation Res 2008;11:151-61.

5. Pilotto A, Gallina P, Fontana A, et al. Development and validation of a multidimensional prognostic index for mortality based on a standardized multidimensional assessment schedule (MPISVaMA) in community-dwelling older subjects. J Am Med Dir Assoc 2013;14:287-92.

6. Angleman SB, Santoni G, Pilotto A, et al. Multidimensional Prognostic Index in Association with Future Mortality and Number of Hospital Days in a Population-Based Sample of Older Adults: Results of the EU Funded MPI_AGE Project. PLoS One 2015.

7. Pilotto A, Sancarlo D, Panza F, et al. Multidimensional Prognostic Index based on a comprehensive geriatric assessment predicts short-term mortality in older patients with heart failure. Circ Heart Fail 2010;3:191-9.

8. Pilotto A, Sancarlo D, Aucella F, et al. Addition of the multidimensional prognostic index to the estimated glomerular filtration rate improves prediction of long-term all-cause mortality in older patients with chronic kidney disease. Rejuvenation Res 2012;15:82-8.

9. Brunello A, Fontana A, Zafferri V, et al. Development of an oncological-multidimensional prognostic index (OncoMPI) for mortality prediction in older cancer patients. J Cancer Res Clin Oncol 2016;142:1069-77.

10. Gallucci M, Battistella G, Bergamelli C, et al. Multidimensional Prognostic Index in a Cognitive Impairment Outpatient Setting: Mortality and Hospitalizations. The Treviso Dementia
(TREDEM) Study. J Alzheimer's Dis 2014;42:1461-8.

11. Amanzio M, Palermo S, Zucca M, et al. Neuropsychological correlates of prefrailty in neurocognitive disorders: a possible role for metacognitive dysfuntion and mood changes. Front Med 2017;4:199.

12. Maggi S, Noale M, Pilotto A, et al. The METABOLIC Study: Multidimensional assessment of health and functional status in older patients with type 2 diabetes taking oral antidiabetic treatment. Diabetes Metab 2013;39:236-43.

13. Pilotto A, Veronese N, Quispe Guerrero KL, et al. Development and Validation of a Self-Administered Multidimensional Prognostic Index to Predict Negative Health Outcomes in CommunityDwelling Persons. Rejuvenation Res 2018 [Epub ahead of print]

14. Barreto PdS, Greig C, Ferrandez AM. Detecting and categorizing frailty status in older adults using a self-report screening instruments. Arch Gerontol Geriatr 2012;54:e249-54.

15. Mantwill S, Fiordelli M, Ludolph R, et al. EMPOWER-support of patient empowerment by an intelligent selfmanagement pathway for patients: study protocol. BMC Med Inform Decis Mak 2015;15:18.

16. von Elm E, Altman DG, Egger M, et al. The strengthening the reporting of observational studies in epidemiology (STROBE) statement: Guidelines for reporting observational studies. J Clin Epidemiol 2008;61:344-9.

17. Tan SS, Pisano MM, Boone AL, et al. Evaluation design of EFFICHRONIC: The Chronic Disease Self-Management Programme (CDSMP) Intervention for Citizens with a low socioeconomic position. Int $\mathrm{J}$ Environ Res Public Health 2019;16:pii:E1883.

18. Mahoney FI, Barthel DW. Functional evaluation: The Barthel Index. Md State Med J 1965;14:61-5.

19. Katz PP; for the association of Rheumatology Health Professional Outcomes Measures Task Force. Measures of adult general functional status: The Barthel Index, Katz Index of activities of daily living; health assessment questionnaire (HAQ), MACTAR patient preference disability questionnaire, and modified health assessment questionnaire (MHAQ). Arthritis Care Res 2003;49(S5):S15-27.

20. Lawton MP, Brody EM. Assessment of older people: Self-maintaining and instrumental activities of daily living. Gerontologist 1969;9:179-86.

21. Brown J, Pengas G, Dawson K, et al. Self-administered cognitive screening test (TYM) for detection of Alzheimer's disease: Cross sectional study. BMJ 2009;338:b2030.

22. Donini LM, Marrocco W, Marrocco C, Lenzi A. Validity of the self-mini nutritional assessment (SELF-MNA) for the evaluation of nutritional risk. A crosssectional study conducted in general practice. J Nutr Health Aging 2018;22:44-52.

23. Linn BS, Linn MW, Gurel L. Cumulative illness rating scale. J Am Geriatr Soc 1968;16:622-6.

24. Garcia-Caselles P, Miralles R, Arellano $M$, et al. Validation of a modified version of the Gijon's social familial evaluation scale (SFES): The "Barcelona SFES Version", for patients with cognitive impairment. Arch Gerontol Geriatr Suppl 2004;9:201-6.

25. Pilotto A, Sancarlo D, Daragjati J, Panza F. Perspective: The challenge of clinical decision-making for drug treatment in older people. The role of multidimensional assessment in older people. Front Med 2015;1:61.

26. Nunes DP, Duarte YAdO, Santos JLF, Lebrão ML. Screening for frailty in older adults using a self-reported instrument. Rev Saúde Pública 2015;49:2.

27. Pilotto A, Rengo F, Marchionni N, et al. Comparing the prognostic accuracy for all-cause mortality of frailty instruments: A multicentre 1-year follow-up in hospitalized older patients. PLoS One 2012; 7:e29090.

28. Angleman SB, Santoni G, Pilotto A, et al. Multidimensional Prognostic Index in Association with Future Mortality and Number of Hospital Days in a Population-Based Sample of Older Adults: Results of the EU Funded MPI_AGE Project. PLoS One 2015;29;10:e0133789.

29. Meyer AM, Siri G, Becker I, et al. The Multidimensional Prognostic Index in General Practice: one-year follow-up study. Int J Clin Pract 2019; 13403. [Epub ahead of print]

30. Bagshaw M, Majumdar SR, Rolfson DB. A prospective multicenter cohort study of frailty in younger critically ill patients. Crit Care 2016;20:175. 